\title{
Récits, figures et chemins de traverse
}

Figures, narratives and byways

Delphine Saurier et Odile Vallée

\section{(2) OpenEdition}

Journals

Édition électronique

URL : https://journals.openedition.org/questionsdecommunication/11152

DOI : 10.4000/questionsdecommunication. 11152

ISSN : 2259-8901

\section{Éditeur}

Presses universitaires de Lorraine

\section{Édition imprimée}

Date de publication : 1 septembre 2017

Pagination : 279-290

ISBN : 9782814303256

ISSN : 1633-5961

\section{Référence électronique}

Delphine Saurier et Odile Vallée, «Récits, figures et chemins de traverse », Questions de communication [En ligne], 31 | 2017, mis en ligne le 01 septembre 2019, consulté le 26 octobre 2021. URL : http:// journals.openedition.org/questionsdecommunication/11152; DOI : https://doi.org/10.4000/ questionsdecommunication. 11152 


\section{$>$ ÉCHANGES}

DELPHINE SAURIER

SciencesCom

Audencia Nantes School of Management

F-44000

dsaurier@audencia.com

ODILE VALLÉE

SciencesCom

Audencia Nantes School of Management

$\mathrm{F}-44000$

ovallee@audencia.com

\section{RÉCITS, FIGURES ET CHEMINS DE TRAVERSE}

Résumé. - À partir de recherches portant sur la notion de figure, les auteures proposent une réflexion sur la place que peut avoir le récit et la narratologie en sciences de l'information et de la communication, et inversement. De la rencontre du récit sur le terrain des processus de communication au concept de récit pris dans son sens anthropologique, c'est une réflexion sur la fabrique de la recherche qui est proposée. De cette réflexion, il semble qu'une manière pertinente d'améliorer la qualité de la recherche dans le domaine de la narratologie passe par une pollinisation par et dans différentes disciplines, suggérant d'éviter la tentation de l'institutionnalisation.

Mots clés. - récit, figure, interdisciplinarité, terrain, épistémologie 
S oyons claires : à la première lecture, il nous est apparu que l'article de Raphaël Baroni (20 l 6) «L'empire de la narratologie,ses défis et ses faiblesses » embrassait avec précision la question de la narratologie, jusqu'à saturation'. Que dire, après cette lecture, sur le récit qui prend une place importante - parfois centrale - dans nos explorations scientifiques? Mais surtout, que dire de ce texte qui nous place dans la catégorie des « simples usagers » (ibid. : 227) certainement maladroits de la « boîte à outils » (ibid. : 220) de la narratologie ? Comment discuter le texte quand il renvoie dos à dos les « simples usagers » de la narratologie et les « narratologues » assumés ou qui s'ignorent? Le sujet aimante : retournons à la lecture de l'article pour nous concentrer sur la démonstration argumentée et référencée. Elle suscite un échange vif entre nous. « Récit », « narration », « narratologie »... « théorie du récit » : les concepts - auxquels nous tentons de nous raccrocher - recèlent une clarté sous-entendue dans le propos, sans pour autant être définis, présentant alors une proximité d'usage troublante. Nous constatons que des usages du récit, que nos recherches nous ont amenées à fréquenter, manquent étonnamment à l'état de l'art ${ }^{2}$. La partition entre « discipline institutionnelle » et « discipline de recherche » (ibid. : 230) nous apparaît comme un point clé. Si cette distinction de Raphaël Baroni, reprise de Dominique Maingueneau, est vérifiable et vérifiée, la logique du mariage interroge néanmoins lorsque l'auteur évoque la narration comme une « métadiscipline » (ibid.). La porte s'entrouvre à la lecture du passage suivant :

« En résumé, pour les chercheurs qui fusionnent généralement une conception pro-narrativiste avec une épistémologie constructiviste [...], nos identités seraient nécessairement le produit d'une médiation narrative et notre rapport au monde dépendrait d'une mise en intrigue transformant la réalité, qui serait à l'origine informe et muette, soumise à un flux temporelle dépourvu de structure ou de signification » (ibid. : 223).

Elle laisse entrer un questionnement réflexif concernant notre rapport au terrain, à l'objet de recherche, à l'angle d'observation, à la discipline. Nous attraperons les fils de la démonstration en tant que chercheures en sciences de l'information et de la communication (SIC), attentives aux processus par lesquels les objets de l'attention évoluent. C'est de cette pratique située que nous pouvons - et proposons de - regarder les propos conclusifs de Raphaël Baroni sur l'importance d'institutionnaliser la narratologie.

\section{Du problème institutionnel de la narratologie}

Revenons à la démonstration de Raphaël Baroni, telle que nous l'avons comprise. L'auteur part du constat que la narratologie est moribonde d'un point de vue institutionnel, précisant que « si la narratologie possède encore un territoire, ce ne peut être qu'une réserve ou un hospice, situés dans les placards de départements

\footnotetext{
' Nous tenons à remercier ici Joëlle Le Marec pour sa lecture et ses précieux conseils.

${ }^{2}$ La notion de récit est diversement investie par les théoriciens des organisations, les anthropologues et les historiens.
} 
de littérature eux-mêmes agonisants » (ibid. : 22I). Pourtant, le territoire social du récit est toujours plus grand, tel un empire capable d'« envahissement » ou d'« asservissement » (ibid. : 222), soumettant, selon certains chercheurs, toutes les autres matrices explicatives de la réalité sociale. État de fait engendré par l'impact des récits sur la réalité, que d'aucun ne peut nier, accompagné par « une ambivalence éthique de l'usage des récits » (ibid. : 223) et personnifié par l'homo fabulator. Les analyses portant sur ce dernier montrent qu'il est capable de fabulation, acte assurant l'épanouissement de la personnalité humaine et d'une identité collective. Raphaël Baroni rappelle ici, avec l'appui de James Phelan, qu'il s'agit de bien distinguer le récit comme production culturelle des compétences cognitives qui en sont l'origine ou qui en permettent l'interprétation. Une distinction, ajoutonsnous, que proposait déjà Marcel Proust (1954 : I I0) un siècle auparavant, lorsqu'il envisageait le pouvoir du romancier:

\begin{abstract}
« Nous sommes tous devant le romancier comme les esclaves devant l'empereur: d'un mot, il peut nous affranchir. Par lui, nous perdons notre ancienne condition pour connaître celle du général, du tisseur, de la chanteuse, du gentilhomme campagnard, la vie des champs, le jeu, la chasse, la haine, l'amour, la vie des camps. Par lui, nous sommes Napoléon, Savonarole, un paysan, bien plus - existence que nous aurions pu ne jamais connaître - nous sommes nous-même. II prête une voix à la foule, à la solitude, au vieil ecclésiastique, au sculpteur, à l'enfant, au cheval, à notre âme. Par lui nous sommes le véritable Protée qui revêt successivement toutes les formes de la vie. À les échanger ainsi les unes contre les autres, nous sentons que pour notre être, devenu si agile et si fort, elles ne sont qu'un jeu, un masque lamentable ou plaisant, mais qui n'a rien de bien réel. Notre infortune ou notre fortune cesse pour un instant de nous tyranniser, nous jouons avec elle et avec celle des autres. C'est pourquoi en fermant un beau roman, même triste, nous nous sentons si heureux ».
\end{abstract}

Si le récit s'immisce dans les questionnements développés par de nombreuses disciplines, Raphaël Baroni (2016 : 226) dénonce une pauvreté de l'usage de la narratologie par les chercheurs de ces autres disciplines : il se réduit trop souvent à l'emprunt de « concepts importés, souvent datés, qui fonctionnent comme une terminologie stabilisée, dans l'ignorance presque totale des travaux entrepris dans le domaine de la théorie du récit au cours des 30 dernières années ». Si la nature trop répandue de « boîte à outils » de la narratologie ne fait aucun doute, pourtant, Raphaël Baroni souligne qu'il existe « au sein de la communauté francophone de nombreux chercheurs qui continuent de faire progresser la compréhension de la narrativité dans ses diverses manifestations, médiatiques ou culturelles, au lieu de se contenter de fonder leur réflexion sur une théorie standardisée et simplifiée » (ibid. : 228). Comment expliquer ce contexte global impropre à une contribution positive à la théorie du récit?

Raphaël Baroni estime que ce contexte s'explique avant tout par un problème d'institutionnalisation de la narratologie dans le paysage académique. L'auteur, suivant la distinction de Dominique Maingueneau (2006: $§ 3$ ), analyse que si la narratologie comme discipline de recherche est active et pertinente, la narratologie comme discipline institutionnelle a tout à construire. Mais « nous sommes [. . . ] face à un cercle vicieux : on peut faire de la narratologie depuis de nombreux lieux institutionnels, et il est évident qu'une certaine interdisciplinarité est requise pour faire avancer cette "métadiscipline", mais on a de plus en plus de mal à situer le lieu où se situe la théorie du récit, 
et donc de suivre ses débats, ses évolutions, ou de s'identifier à ses enjeux » (ibid. : 230). La solution est de trois ordres. Idéale et idéaliste, il s'agirait de la création d'une nouvelle section au Conseil national des universités (CNU). Performante mais difficile, la solution serait la création de lieux institutionnels comme des séminaires et surtout des chaires. Efficace et réalisable, la narratologie pourrait acquérir une réelle visibilité au sein d'une autre discipline comme les études littéraires, les sciences du langage et les sIc.

\title{
Les motivations à institutionnaliser: entre nécessité scientifique et désirs individuels
}

Cet appel de Raphaël Baroni pour plus de visibilité de la narratologie sur la scène scientifique peut rappeler des démarches similaires, comme celles des sciences de l'éducation et encore des SIC, respectivement sections 70 et 7 I au cNU. Remontent également à l'esprit les nombreux débats concernant l'institutionnalisation de la criminologie, qui se condensent relativement bien dans la présentation suivante d'une livraison de Cultures et Conflits (Bigo, Bonelli, 2014) :

\begin{abstract}
« Pour le sociologue ou le politiste français, l'expression même de "criminologie" sonne au premier abord comme un peu étrange et un peu ridicule. Pourquoi séparer des comportements - criminels en l'occurrence - de l'ensemble des relations sociales dans lesquelles ils sont encastrés et en faire un champ d'étude spécifique? Si la criminologie se focalise seulement sur le crime, elle n'a guère plus de sens qu'une "anorexicologie", une "suicidologie" ou une "mariagologie", respectivement entendues comme sciences de l'anorexie, du suicide et du mariage. Si, en revanche, son ambition est de resituer le crime dans la fabrication et le maintien d'un ordre social et politique, pourquoi la distinguer de la sociologie ou de la science politique ? La question est volontairement naïve. Selon les pays, l'existence ou non de la criminologie comme discipline, de même que le sens qui y est donné dépendent de trajectoires historiques singulières dont il faut à chaque fois retracer les étapes et les modalités. Les rapports de forces sociaux et les équilibres académiques entre le droit, la sociologie, la médecine et la psychologie dessinent en effet des formes d'institutionnalisation différentes au Canada, en Belgique, aux États-Unis, au Royaume-Uni, en Allemagne, en Italie ou en France. Toutefois, la naïveté de la question n'est qu'apparente. Les batailles pour l'existence disciplinaire de la criminologie demeurent en effet inséparables d'une promotion plus large de modes de questionnement, de catégories de pensée et de mise en récit spécifiques, c'est-à-dire d'une "raison criminologique" que ce numéro spécial de Cultures \& Conflits entend interroger ».
\end{abstract}

On retrouve dans cette virulente introduction une majorité des axes de réflexion dégagées par Raphaël Baroni pour la narratologie, la dénonciation en moins. D'abord, le récit et le crime sont omniprésents sur la scène sociale et politique. Comme sujets et formes communicationnelles, ils sont présents dans les paroles, l'environnement, les représentations, le rapport aux autres. Cette omniprésence se retrouve dans des croyances et des jugements moraux, qui offrent une emprise de la question criminelle et du récit sur les décisions politiques et sociales.

Ensuite, Raphaël Baroni insiste sur la difficulté de rendre visibles la narratologie et ses apports, ce qui entraîne notamment sa réduction au caractère de « boîte à outils »). De la même façon que le récit concerne plusieurs disciplines, le crime est traité par le droit, la sociologie, la médecine et la psychologie - au moins -, ce qui 
conduit à un manque de visibilité des savoirs produits sur le sujet... Un regret peut alors étreindre le chercheur à l'idée que ces savoirs, par manque de visibilité, ne participeront pas - ou peu - à une évolution raisonnée de la société.

Pour continuer, la constitution d'une section cNu est envisagée par Raphaël Baroni comme la solution : «Dans un monde idéal, il serait probablement plus efficace de créer une nouvelle section » (Baroni, 2016 : 235), quand elle est effective quelques mois seulement de l'année 2012 pour la criminologie. On le voit en actes pour la criminologie et en paroles avec Raphaël Baroni : cette reconnaissance institutionnelle pose la question de la légitimité scientifique des chercheurs occupant la place et de leur désir de reconnaissance institutionnelle, voire politique. Raphaël Baroni, à cet égard, distingue « les véritables théoriciens de cette forme particulière de communication » des « simples usagers » (ibid. : 227). Pourquoi et pour qui se lancet-on dans une telle proposition, qui apparait plutôt sous la forme d'une revendication dans le cas de la criminologie ? Prégnance du pour qui dans la polémique française concernant la criminologie. Si le pour qui n'est pas absent de l'article de Raphaël Baroni, le pour quoi est essentiel : éprouver la théorie du récit. D'autant plus que si la criminologie a acquis une reconnaissance institutionnelle dans certaines régions du monde scientifique, il n'en est rien pour la narratologie, démontre l'auteur.

\section{Pour une perspective alternative : comment le récit vient-il aux chercheurs?}

Ce détour comparatiste met en lumière les motivations individuelles et les enjeux sociaux, politiques, scientifiques de la reconnaissance par le cNu. Ce faisant, il redensifie la bipartition de la discipline proposée par Dominique Maingueneau et qui étaye le propos de Raphaël Baroni. En effet, si la distinction entre la « discipline scientifique » et la « discipline institutionnelle » est intellectuellement admise par les chercheurs, ceux-ci l'expérimentent dans la rencontre avec leurs objets et terrains de recherche. Comment distinguer alors la réflexion sur l'objet des dispositifs institutionnels qui la cadrent? Une liste des revues reconnues par la section CNU, une composition du comité scientifique de la revue, un financement européen, un champ disciplinaire structuré par des acteurs, des concepts, des méthodes : la présence institutionnelle se trouve à tous les moments de la démarche du chercheur, en amont comme en aval, rendant le partage avec la « discipline scientifique » difficile à réaliser. D'autant plus que « les acteurs sont inévitablement portés à croire que de tels découpages [par disciplines] correspondent à une partition effective du réel, à projeter dans quelque transcendance les principes de classement qui structurent leurs pratiques » (Maingueneau, in : Baroni, 2016 : 229).

C'est pourquoi il semble envisageable de développer une deuxième perspective à côté de celle de Raphaël Baroni qui propose que « la meilleure manière d'améliorer la visibilité et la qualité de la recherche et de la formation dans ce domaine passe par une institutionnalisation de certains de ses acteurs, même si des chercheurs 
appartenant à des horizons divers continueront (et devraient continuer), chacun à partir de son point de vue, à participer au développement de cette théorie générale » (ibid. : 234). Déplacer la focale vers une perspective alternative, contraint toutefois à ne traiter qu'une partie de la proposition de Raphaël Baroni ${ }^{3}$ : la meilleure manière d'améliorer la qualité de la recherche dans ce domaine passe par une pollinisation par et dans différentes disciplines, suggérant d'éviter la tentation de l'institutionnalisation. Le foisonnement observé et concédé par Raphaël Baroni peut avoir pour foyer l'intersection avec d'autres disciplines, y compris sur d'autres objets que l'objet initial du récit écrit. Si l'idéal est réalisé - la création d'une section -, la narratologie ne courtelle pas le risque de se voir progressivement figer par les pratiques de chercheurs ayant intériorisé les structures disciplinaires? Dans quelle mesure créer une nouvelle section - suivant le vœu de Raphaël Baroni - ne conduirait-il pas à se couper de ce foisonnement, à écarter les espaces de croisement productifs et créatifs ? Ceux-là même qui participent à garantir aujourd'hui le développement de la théorie du récit...

Cela pose la question fondamentale, épistémologique et méthodologique, de la fabrique de la recherche. Comment le récit vient-il au chercheur en sIC que nous sommes? Comment s'imbrique-t-il dans nos démarches info-communicationnelles? Nous nous sommes intéressées au récit par le biais des acteurs et des usages: dans ce cas, le récit devient un élément constitutif de l'objet de recherche et non plus un concept exogène et surimposé. Le récit s'est imposé comme l'un des éléments de l'analyse de phénomènes communicationnels et l'on a cherché à le comprendre dans ses dimensions à la fois logistique, poétique et sociale, à le saisir dans sa vie triviale (Jeanneret, 2008). Pour ce faire, nous avons effectivement opéré un usage fonctionnel des conceptualisations issues de la narratologie classique et postclassique, pour reprendre l'opposition introduite par David Herman (1997), complétées par un corpus théorique issu de la sociologie, de la sémiologie et des sciences de l'information et de la communication (sIC).

De plus, s'observe également en sIC une approche du récit, non plus comme un matériau tangible, circonscrit, mais comme un concept qui contribue à répondre à notre prétention à l'analyse du processus de communication. Le récit est donc aussi pensé en sIC comme un élément « méta » qui vient lisser le processus de communication. En effet, il tend à rendre transparentes les médiations qui résident au cœur du processus. Jean-Michel Adam ( 1984 : I I), linguiste « postclassique » (Baroni, 2016 :226), qui s'intéresse à l'analyse textuelle des discours, décrit l'acte de discours qu'accomplit le récit de la façon suivante : « Le régime normal (classique) du récit repose sur le déni des opérations de productions (codage, montage), sur l'oubli qu'une instance organise la représentation et en règle la lecture ».

Sous ces auspices, le récit compris dans le processus de communication - et non plus dans sa seule fonction de « boîte à outils »-a pour effet de lisser et harmoniser,

\footnotetext{
${ }^{3}$ On remarquera que nous évacuons la question de la visibilité et celle de la formation.

${ }^{4}$ Le préfixe « méta » est compris autant dans son sens de dépassement et d'approfondissement que dans son sens scientifique usuel d'autoréférence.
} 
il produit cette « identité narrative » controversée, critiquée par Raphaël Baroni. Sa contextualisation dans des lieux et des pratiques sociales et sa déconstruction permettent donc de questionner finement le processus de communication, acte rendu d'autant plus crucial dans un contexte où les médiations sont fortement redéfinies par le numérique et par l'idéologie du lien direct. Ici, le niveau d'appropriation du concept de récit est en réalité celui du narratif et de la narrativité, compris comme ce qui fait advenir le récit. Considérant ainsi le récit, le chercheur est à même de questionner ses formes et frontières, ainsi que son échelle d'observation. Cela permet aussi de saisir le processus et les effets de l'articulation, délibérée ou fortuite, d'un assemblage hétéroclite de médias, de discours, de textes, d'images, de pratiques, de figures, parfois distants dans le temps et dans l'espace. Cela permet enfin de remettre au cœur de la réflexion l'idée de la tension narrative qui participe à la structuration du processus de communication, qui accompagne les formes culturelles dans leur vie triviale, qui garantit in fine leur pérennité sociale.

Insérer le récit et la narrativité au cœur de l'analyse du processus de communication rend aux actions leur épaisseur symbolique, aux dispositifs et aux structures leur épaisseur historique et sociale, à l'irrationnel sa présence ; en même temps que la tension narrative garantit l'ouverture des possibles.

\section{La figure, le récit et les SIC}

Comment cette perspective se met-elle concrètement en place ? En partant toutes deux de questionnements différents, nous nous sommes intéressées à des figures, et plus précisément aux modalités de leur émergence et de leur évolution. Qu'est-ce qui fait l'opérativité symbolique (Quéré, 1992) du microcrédit comme une cause (Voirol, 2003) ? L'analyse du discours des acteurs de cette cause conduit à s'interroger sur l'émergence de deux figures complémentaires (Vallée, 2014) : celle du microentrepreneur et celle du macro-entrepreneur ${ }^{5}$. La construction de ces figures repose sur différents récits et productions discursives : des portraits visuels et narratifs, des biographies, des autobiographies, des médias personnels, des allocutions, des DVD, des vidéos, des rapports d'activités, etc. Autres espaces, autres figures : en quoi les visiteurs contribuent-ils au surgissement (Hennion, 1993) - toujours renouvelé et toujours à venir - d'un lieu de mémoire (Nora, 1984) ? L'analyse conjointe de la patrimonialisation

\footnotetext{
${ }^{5}$ La Campagne du Sommet du microcrédit est un réseau international qui rassemble des acteurs hétérogènes du secteur de la microfinance - secteur qui délivre des prêts et produits financiers a des personnes « paurres » et/ou exclues des liens financiers formels. Le discours du réseau est le lieu depuis lequel est identifiée et analysée l'émergence des figures. Créé en 1997, le réseau travaille à la reconnaissance du microcrédit comme un outil efficace de lutte contre la pauvreté à travers des actions de terrain et une démarche de plaidoyer et de communication stratégique menée auprès des décideurs institutionnels et politiques et auprès du grand public. Deux figures structurent la rhétorique du réseau : le miro-entrepreneur, « héros » ordinaire, incarné par les femmes pauvres emprunteuses, enjeu de la démarche de microcrédit et le macro-entrepreneur, incarné par des leaders engagés, fondateurs d'institutions de microfinance emblématiques, « héros » extra-ordinaire, moteur et garant de l'orientation éthique de la démarche.
} 
de lieux consacrés à Marcel Proust, Marie et Pierre Curie et Frédéric et Irène JoliotCurie et de la réception des visiteurs a permis d'identifier la figure de la personne célèbre - autrement labellisée aujourd'hui illustre - comme l'une des balises du surgissement des lieux de mémoire. Cette figure résulte de l'imbrication de différentes productions discursives et récits : des biographies, des essais littéraires, des allocutions politiques, des discours commémoratifs, la scénographie des lieux, des récits de vies illustres véhiculés dans l'espace public, etc. Autrement dit, la figure apparaît comme un objet incontournable à analyser afin d'envisager nos problématiques respectives se rattachant à la patrimonialisation et à la sociologie de la réception pour l'une, à la circulation des problèmes publics pour l'autre. Dans ce cadre, on se rend compte de la prégnance du récit et de la façon dont il participe à l'émergence et au modelage continu de la figure. Le récit s'impose donc d'abord comme une des catégories des matériaux des recherches.

Le point de départ de l'analyse narrative est donc empirique et doit permettre de saisir des figures dans leur circulation sociale et leurs raisons politiques. Notons que le récit trouve sur ces deux terrains un statut similaire, quoique à des degrés différents : s'il est toujours omniprésent, il est revendiqué par les acteurs de la promotion du microcrédit ${ }^{6}$ et convoqué par les acteurs des lieux de mémoire. Les récits et leurs usages s'étalent dans le temps et revêtent des formes variées, constituant alors des corpus de recherche composite. Les figures de Marcel Proust, Marie et Pierre Curie et Frédéric et Irène Juliot-Curie émergent de l'exploration des créations et de la vie même des illustres qui offrent matière à récits (Elias, 1991) ; des paroles des illustres qui se sont racontés dans l'espace public pour y construire une posture (Meizoz, 2007 ; Saurier, 20I I) ; des histoires portées par les critiques littéraires (Jeanneret, 1982) et le travail des experts (Heinich, 199I) qui offrent un cercle rapproché de légitimation de la figure ; des appropriations politiques et communautaires (Saurier, 20I3) qui mêlent intimement leur récit propre à l'histoire des illustres, permettant de faire circuler la figure dans des cercles sociaux plus éloignés ; des récits patrimoniaux et de l'interprétation des visiteurs (Heinich, 199I ; Saurier, 2013) qui permettent aux figures des illustres de rejoindre la mémoire collective. Corpus de recherche composite encore lorsque l'on se penche sur la figure émergente du macro-entrepreneur, dont Muhammad Yunus, réputé le fondateur du microcrédit est un exemple très médiatisé, qui répond à celle du micro-entrepreneur. Les modalités d'apparition de l'initiateur des projets de microcrédit sur le terrain et cheville ouvrière de son développement sont variées et complexes. II prend la parole en son nom propre et développe une thétorique qui alimente et excède celle du réseau d'acteurs depuis lequel elle est analysée. II fait l'objet d'hagiographies. Finalement, l'analyse des récits montre que leurs usages participent à

« une $[\ldots]$ dynamique $[\ldots]$ qui pousse les vivants à devenir des signes, à trouver dans un discours le moyen de se transformer en une unité de sens, en une identité. De cette chair opaque et dispersée, de cette vie exorbitante et trouble, passer enfin à la limpidité d'un mot, devenir un fragment du langage, un seul nom, lisible par d'autres, citable [...] » (Certeau, 1990 : 217-218).

\footnotetext{
${ }^{6}$ L'analyse s'est précisément centrée sur le discours de la Campagne du Sommet du Microcrédit, un
} réseau international d'acteurs de la microfinance fortement engagé dans la promotion du microcrédit. 
C'est ainsi que les entrelacs de récits et de productions discursives analysés forment des « ferments narratifs » et font émerger un nouveau « récit » lisible, citable, mémorisable, mémorable et incarné par la figure. Car les modes d'apparition et d'expression du macro-entrepreneur comme ceux des illustres s'appuient à la fois sur du narratif et de la narrativité que Philippe Marion (1997 : 84) distingue de la façon suivante :

« II faudrait donc différencier le narratif, comme état explicite et affirmé, du narratif comme dimension possible, au vu d'une certaine configuration de l'objet observé (qu'il soit un signe, un message, mais aussi plus fondamentalement, un média). La narrativité serait non seulement un résultat mais elle contiendrait aussi une dimension promissive, celle d'un potentiel ou d'un développement virtuel suggérés par la présence simultanée de certains indices ».

C'est en analysant des récits hétérogènes à la fois dans leur matérialité, leur historicité et leur puissance narrative, énoncés par différents acteurs et depuis différents espaces, que nous saisissons la force des figures. Cette démarche analytique permet de voir comment les figures se chargent de valeurs pour atteindre tantôt des points de rupture ${ }^{7}$, tantôt les sphères de la consécration : autrement dit, elles peuvent être transfigurées mais aussi défigurées par l'usage rhétorique et les effets de leur circulation et de leurs appropriations. Les valeurs qu'elles portent font sens social et les figures offrent à la société un récit d'elles-mêmes toujours singulier. La force de ce récit réside dans sa tension narrative : le « nouement »(Baroni, 2007: 402) initial de l'intrigue (le rejet de Vincent van Gogh, l'homosexualité de Marcel Proust, la découverte scientifique par une femme pour Marie Curie, la posture contre-intuitive de Muhammad Yunus, la capacité de gestion financière des femmes pauvres) trouve son dénouement dans la reconnaissance et la mémoire collectives. Mais si ces figures perdurent avec autant de vivacité, on estime que c'est en raison d'un dénouement résolu collectivement, mais surtout partiellement. En effet, les récits analysés (fiction, discours commémoratifs, scénographie...) offrent une cohérence globale dans un espace-temps, mais ils présentent aussi des aspérités à l'interprète : s'ils racontent une même histoire, leur narration est toujours singulière. Ce sont ces aspérités qui interrogent l'interprète, qui continuent à nouer l'intrigue autour d'une figure que l'interprète comprend comme objet impossible à saisir dans son entièreté. Le récit du créateur (de son génie et de ses potentialités) que ces figures portent restera toujours énigmatique en raison de la narrativité inachevée qui les fait advenir. Et finalement, comme le souligne Louis Marin (1978 : 8), « cette puissante assurance du récit qu'a son pouvoir de vérité, cette immédiate habilitation de l'histoire à tenir le discours du réel [et qui] a provoqué un soupçon, le soupçon que le récit est aussi un piège et d'autant plus efficace qu'il n'apparaît point tel ».

Ainsi, au terme d'un processus d'appropriations sociales, les figures sont-elles à même de se raconter, leur évocation convoque un récit partagé et vivant dans la mémoire collective. C'est alors une compréhension du récit dans sa dimension anthropologique que nos recherches envisagent. Ces récits contemporains du micro et du macro-entrepreneur, de Marcel Proust et de Marie Curie puisent dans le fonds

\footnotetext{
${ }^{7}$ C'est la trajectoire qu'analyse Y. Jeanneret (1982) concernant Romain Rolland et celle de Frédéric Joliot-Curie analysée par D. Saurier (2013).
} 
commun de la figure du créateur, celle qui, affranchie au moins partiellement des structures qui assurent la reproduction sociale, est capable d'innover, de transformer, de créer. Ces figures de créateurs matérialisent des récits émancipateurs :

«Si notre conception de la réalité est une construction visant à rendre le monde habitable, il importe
qu'elle soit en mesure d'évoluer quand elle révèle ses limites, de s'adapter aux heurts incessants
que lui oppose un univers d'expériences concrètes irréductibles à nos schémas interprétatifs et
comportementaux. L'érosion permanente des normes de la vie quotidienne est aussi nécessaire
et vitale à lindividu et à la société que leur reproduction à travers des processus régulateurs. Les
anecdotes que l'on se raconte, mais surtout les récits de fiction, par leur pouvoir de forger des
mondes possibles inédits, permettent ainsi d'explorer des virtualités insoupçonnées de la réalité, elles
visent alors à nous "défamiliariser" de notre environnement quotidien » (Baroni, 2007: 410).

Nos démarches ont permis d'expérimenter une position inconfortable de laquelle nous semble naître la fécondité. La nature de nos terrains nous a engagées à regarder le récit et la théorie de celui-ci du point de vue très particulier de notre discipline. Cette double position d'extériorité conceptuelle et de légitimité empirique vis-à-vis de la théorie du récit engage à un autre regard sur celle-ci, apportant une réponse située qui appelle à des compléments.

Il est donc évident que nous avons imposé un détour info-communicationnel au récit, l'éloignant de la littérarité initiale. Nos figures admises comme êtres culturels s'offrant en récit dans l'espace public questionnent le rapport entre récit et narrativité. La figure offre un récit qui s'inscrit dans et qui inscrit une culture : il est polyphonique sans unique narrateur ; il est constitué d'une infinité de narrations qui se déploient dans une narrativité continue. Cette dernière s'élabore dans des paroles, dans des formes, dans des structures : il s'agit de l'acte mimétique, la synthèse de l'hétérogène cherchant une configuration du temps (Ricœur, 1983). Si le récit est inclus dans la narrativité, il peut aussi la précéder, la suivre, voire l'excéder : les récits préalables nourrissent la narrativité qui fait advenir le récit sur les figures; les figures résultent de la narrativité comprise comme un processus ${ }^{8}$; enfin, le récit excède la narrativité lorsqu'il s'autonomise en tant qu'être culturel.

\section{Conclusion}

La proposition qualifiée d'idéale par Raphaël Baroni, concernant la création d'une nouvelle section du cNu, nécessite selon nous d'être discutée. Ce que nous avons cherché à faire, partant de nos postures de chercheurs en SIC et à partir d'un objet particulier : la figure. Discuter cette proposition permet de réfléchir au traitement de la question circonscrite de la narratologie comme discipline de recherche et discipline institutionnelle. Au-delà, discuter cette proposition permet de s'interroger sur les dessous de la fabrication des connaissances, plus précisément sur les frontières disciplinaires considérées à partir des terrains. En effet, cela nous

\footnotetext{
${ }^{8}$ Nous pouvons ici proposer une analogie féconde à la compréhension du propos : le récit est à la narrativité ce que le patrimoine est à la patrimonialisation.
} 
a engagé à revenir sur notre façon de construire nos terrains, réfléchis comme des composites au sens développé par Joëlle Le Marec (2002 : 63) :

\begin{abstract}
«Au stade actuel,le concept de composite n'existe pas autrement que comme reconceptualisation de la séparation et de l'articulation entre le terrain comme "unité" complexe organisée par l'approche communicationnelle des phénomènes sociaux et l'objet de recherche construit à travers ce terrain ».
\end{abstract}

L'unité articulée dans et par le processus de communication renvoie à la fois à des lieux de pratiques sociales suggérant la convocation de certains concepts (le récit comme matériau à analyser), et à un lieu reconfiguré par la recherche, borné par des contraintes empiriques et théoriques (le récit et la narrativité discutés comme concepts). C'est une réflexion plus globale sur l'interdisciplinarité qui s'engage, non pas comme un vœu pieu mais comme une réalité scientifiquement construite qu'il s'agit d'analyser aussi dans les processus de communication qui la soutiennent. Concrètement, ce que le récit fait aux SIC, et inversement...

Suivant cette compréhension de la recherche, par le biais du rapport au terrain, nous nous accordons donc particulièrement avec Raphaël Baroni (2016 : 234) lorsqu'il affirme:

« Si la variété des approches et l'interdisciplinarité demeurent une nécessité pour un objet aussi polymorphe dans ses usages que dans ses incarnations médiatiques, il s'agit malgré tout de garantir l'existence d'un lieu institutionnel vers lequel toutes ces perspectives puissent converger [pour] réfléchir à la complémentarité des approches ainsi que [...] mesurer leur impact sur une théorie générale de la narrativité. Seule l'existence de chaire de narratologie garantirait l'essor d'une théorie du récit contemporain viable, en phase avec les enjeux de son époque, et vers laquelle toute personne soucieuse d'intégrer une dimension narrative dans ses pratiques ou sa réflexion pourrait se tourner de manière à se tenir au fait de ses avancées les plus récentes, au lieu de s'en tenir à quelques préjugés ou lieux communs ».

Réfléchissant ainsi, il ne s'agit plus de faire entrer une discipline scientifique dans les logiques d'une discipline institutionnelle. II nous semble que l'une et l'autre ne peuvent et ne doivent pas être raisonnées du point de vue de l'acte de la recherche. Gageons que cette dernière est à même de trouver les chemins de son développement, même lorsqu'il s'agit de chemins de traverse...

\title{
Références
}

Adam J.-M., 1984, Le Récit, Paris, Presses universitaires de France, 1999.

Baroni R., 2007, La Tension narrative. Suspense, curiosité et surprise, Paris, Éd. Le Seuil.

Baroni R., 2016, « L'empire de la narratologie, ses défis et ses faiblesse », Questions de communication, 30, 219-238.

\footnotetext{
9 II s'agit du processus social de communication, mais aussi du processus de communication qui organise la recherche.
} 
Bigo D., Bonelli L., 20 I 4, « Critique de la raison criminologique », Cultures \& Conflits, 94-9596, pp. 7-26. Accès : https://conflits.revues.org// 8879.

Certeau M. de, 1990, L'Invention du quotidien, I. Arts de faire, Paris, Gallimard.

Elias N., 199I, Mozart. Sociologie d'un génie, trad. de l'allemand par J. Etoré et B. Lortholary, Paris, Éd. Le Seuil.

Heinich N., 199I, La Gloire de Van Gogh. Essai d'anthropologie de l'admiration, Paris, Éd. de Minuit.

Hennion A., 1993, La Passion musicale. Une sociologie de la médiation, Paris, Métailié.

Herman D., 1997, « Scripts, Sequences, and Stories: Elements of a Postclassical Narratology », PMLA, vol. I I2, 5, pp. 1046- 1059.

JeanneretY., 1982, Un demi-siècle de réception critique de l'œuvre de Romain Rolland en France ( 1898 - 1945), Thèse en littérature française, Université Sorbonne Nouvelle-Paris 3.

Jeanneret Y., 2008, Penser la trivialité. Volume I : La vie triviale des êtres culturels, Paris, Hermès/Lavoisier.

Le Marec J., 2002, Ce que le " terrain » fait aux concepts. Vers une théorie des composites, Habilitation à diriger des recherches, Paris, Université Paris 7.

Maingueneau D., 2006, « Deux cultures des études littéraires », A Contrario, vol. 4, 2, pp. 8-18. Accès : http://www.cairn.info/revue-a-contrario-2006-2-page-8.htm.

Marin L., 1978, Le Récit est un piège, Paris, Éd. de Minuit.

Marion P., 1997, « Narratologie médiatique et médiagénie des récits 》, Recherches en communication, 7, pp. 6I-88.

Meizoz J., 2007, Postures littéraires. Mises en scène modernes de l'auteur, Genève, Slatkine.

Nora P., dir., 1984, Les Lieux de mémoire, Paris, Gallimard, 1997.

Proust M., 1954, « Le pouvoir du romancier », pp. 109-1 10, in : Proust M., Essais et articles, Paris, Gallimard, 1994.

Quéré L., 1982, Des Miroirs équivoques. Aux origines de la communication moderne, Paris, Aubier Montaigne.

Ricœur P., 1983, Temps et récit, t. I, L'Intrigue et le récit historique, Paris, Éd. Le Seuil.

Saurier D., 201 I, « La figure de l'auteur comme médiation littéraire. L'autoportrait de Marcel Proust », pp. 57-74, in : Alvès A., Pourchet M., dirs, Les Médiations de l'écrivain, Paris, Éd. L'Harmattan.

Saurier D., 2013, La Fabrique des illustres : Proust, Curie, Joliot et lieux de mémoire, Paris, Éd. Non Standard.

Vallée O., 20 I4, Approche communicationnelle de la construction du microcrédit comme cause internationale : pratiques, discours, figures, Thèse en sciences de l'information et de la communication, Université Paris-Sorbonne.

Voirol O., 2003, «Apparition d'une cause politique "globale" et critique de la "mondialisation". L'Action Mondiale des Peuples (AMP) », En ligne : http://www.afsp.msh-paris.fr/activite/ groupe/germm/collgermm03txt/germm03voirol.pdf. 